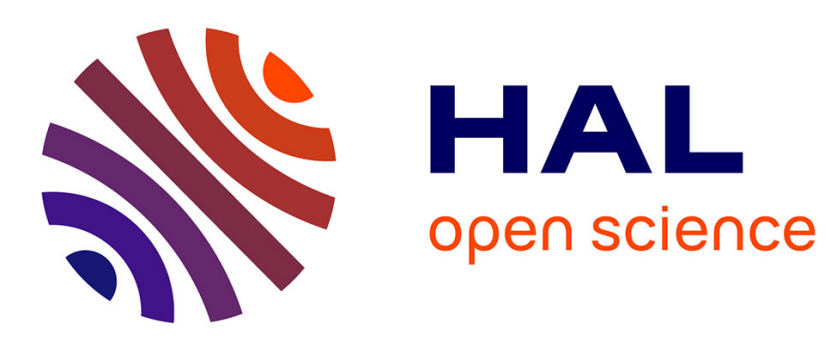

\title{
Tracking the consequences of design decisions in mechatronic systems engineering
}

Pierre Couturier, Mambaye Lo, Abdelhak Imoussaten, Vincent Chapurlat, Jacky Montmain

\section{- To cite this version:}

Pierre Couturier, Mambaye Lo, Abdelhak Imoussaten, Vincent Chapurlat, Jacky Montmain. Tracking the consequences of design decisions in mechatronic systems engineering. Mechatronics, 2014, 24 (7), pp.763 - 774. 10.1016/j.mechatronics.2014.03.004 . hal-00840436

\section{HAL Id: hal-00840436 \\ https://hal.science/hal-00840436}

Submitted on 30 Nov 2018

HAL is a multi-disciplinary open access archive for the deposit and dissemination of scientific research documents, whether they are published or not. The documents may come from teaching and research institutions in France or abroad, or from public or private research centers.
L'archive ouverte pluridisciplinaire HAL, est destinée au dépôt et à la diffusion de documents scientifiques de niveau recherche, publiés ou non, émanant des établissements d'enseignement et de recherche français ou étrangers, des laboratoires publics ou privés. 


\title{
Tracking the consequences of design decisions in mechatronic systems engineering
}

\author{
Pierre Couturier, Mambaye Lô, Abdelhak Imoussaten, Vincent Chapurlat, Jacky Montmain \\ Laboratoire de Génie Informatique et d'Ingénierie de Production (LGI2P) \\ Site de l'Ecole des Mines d'Alès
}

Parc scientifique Georges Besse, 30035 Nîmes cedex 5, France

Corresponding author: Pierre.Couturier@mines-ales.fr, tel: (+33) 466384046

Authors' email: first name.name@mines-ales.fr

\begin{abstract}
:
The design of mechatronic systems involves several technical and scientific disciplines. It is often difficult to anticipate, at the outset, the consequences of design decisions on the ultimate effectiveness of such complex systems, in which case the evaluation process is required to support the designers each time engineering choices must be made or justified. Since designers may belong to different technical and scientific cultures however, their understanding of both the design stakes and the evaluation process is too often biased. Moreover, design choices take place in an uncertain context and according to multiple criteria, some of which may be contradictory. In order to track the consequences of design decisions, we are proposing a conceptual data model to perform evaluations within the MBSE framework of Systems Engineering. We then proceed by relying on the relationships demonstrated by such a model to identify the potential impacts of design choices on future product performance. Since data available during the conceptual phase of the design are typically uncertain or imprecise, an original research protocol is extended to a qualitative impact analysis for the purpose of highlighting the most promising alternative system design solutions (ASDS). An example in the mechatronics field serves to illustrate our proposals.
\end{abstract}

Keywords: Systems engineering, Mechatronics, Evaluation, Traceability.

\section{Introduction}

Mechatronic design is an interdisciplinary activity that continually strives to integrate widespread functionality into geometrically constrained products. In the competitive marketplace, both time and finances are often lacking when it comes to studying and finalizing several mechatronic concepts and then retaining only the most satisfactory one. Engineers therefore require support in reviewing alternative system design solutions (ASDS) and in making and defending the best design choices as of the earliest product design stages [1]. In a Systems Engineering (SE) context [2], such is the role of evaluation activities in assessing ASDS and in ensuring that design-related decisions take into account relevant multidisciplinary knowledge and can hence be duly justified. With this aim, various analytical approaches and methods can be applied to conduct effectiveness, cost and risk studies as well as to compare different ASDS.

The design evaluation process however faces a number of challenges, including:

1) Though the initial choices are definitely critical to ensuring a successful design project, rating the merit factors of each candidate solution during the conceptual design stage is generally subject to uncertainty and inaccuracy; 
2) Designing complex mechatronic products requires multidisciplinary knowledge. Since designers tend to have narrow technical and scientific backgrounds, their understanding of system design objectives and their vision of the evaluation process are often only partial and incomplete;

3) Assessing the consequences when choosing from among several ASDS is a critical step to the process and to this day has still not been resolved effectively [3];

4) System requirements may at times be interpreted as contradictory when considering a given ASDS. The challenge then is to identify satisfactory ASDS that achieve an acceptable balance between these requirements, as opposed to finding the optimal ASDS.

Although the core of design problematic is how to produce solutions, design solutions synthesis is not the scope of the presented research work. This paper aims to provide some basic elements to address the issues raised in 1 through 4 above by considering just the effectiveness evaluation; due to constraints placed on the paper's length, risk and cost aspects will not be included.

After defining the role of evaluation within the design process, Section 2 will tackle the $2^{\text {nd }}$ issue raised above in proposing a conceptual data model considered herein as the abstract syntax of a possible language dedicated to evaluation within the Systems Engineering (SE) framework. The intention is for members of a multidisciplinary design team to be capable of sharing a common vision of data as a prerequisite to evaluating ASDS, regardless of their profession, objectives or specialization. Based on this proposed common view, Section 3 will contribute to resolving issue 3 by means of facilitating the identification of potential impact relations between design choices and effectiveness criteria. Once potential impacts have been identified, the consequences of such impacts on the degree of ASDS satisfaction in a multi-criteria context must be analyzed. To this end, Section 4 will formalize the interactions proposed in this research work between a behavior model of the system being designed and a model of stakeholders' expectations. Our proposal seeks to address issue 4 above by adapting a formal approach to determine the level of criterion satisfaction, which entails applying qualitative or quantitative reasoning depending on the uncertainty inherent in the design specification. Section 5 will illustrate our proposals through an example of developing the electrical assistance function for a wheelchair. Section 6 will draw a conclusion regarding future perspectives.

\section{Evaluation in design}

Let's consider, without the ambition of achieving completeness, a number of current design theories and methodologies that deal with evaluation issues [4,5,6,7]. According to [8], designers are making progress towards defining the systems under design in more concrete terms by iteratively performing the steps indicated in Fig. 1:

- Synthesis: The creative activity by which known elements are placed together in new and more useful combinations in order to produce ASDS;

- Analysis: Deriving an estimation and prediction of design parameter values;

- Evaluation: Comparison of each ASDS with other ASDS and verification of compliance with customer requirements.

Mechatronic engineering combines mechanical engineering, electrical engineering and computer science within an interactive way. The VDI2206 guideline recommends conducting the design of mechatronics systems according to the so-called "V-model" pattern [9]. The design process distinguishes between the problem solving process of the individual designer (micro-level) and the generic process related to design phases (macro-level). At the macro-level the system is specified functionally, working principle variants are evaluated and selected. Then sub-parts allocated to each 
involved discipline such as mechanics, electronics, and computer science are specified, realized and integrated to form a system.

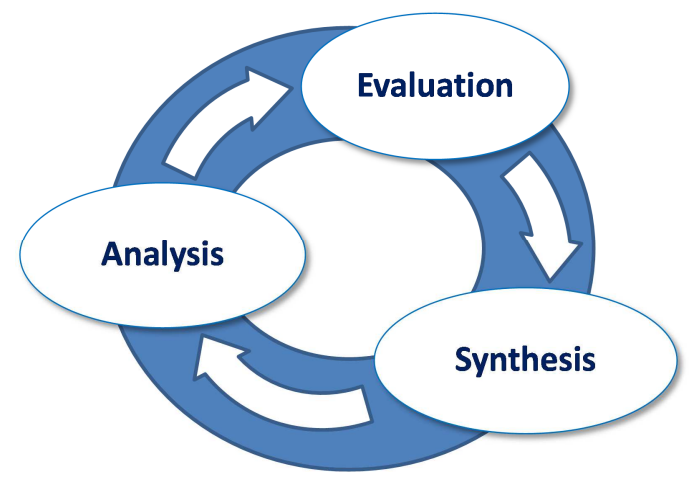

FIGURE 1: Evaluation vs. analysis and synthesis [9]

Several passes through the v-shaped model are necessary to obtain a mature product. Micro-level problem-solving activities are being performed during the design process to generate and then to assess candidate solutions. The present work will distinguish between the evaluation of ASDS (at the macro level) and the evaluation of the design ideas generated at the micro level (during the synthesis step of Fig. 1) in order to produce acceptable solutions (ASDS).

The Function-Behavior-Structure approach supports systematic modeling and reasoning in the systems designing [10] and attempts to explain the act of generating design solutions (thus at the synthesis step of Fig. 1). The Function-Behavior-Structure activities include: a formulation which transforms functions into a set of expected behaviors; a synthesis of a structure which exhibits the expected behavior; an analysis of the behavior produced by the structure; an evaluation between the expected and the produced behavior.

The Function-Behaviour-State modeler provides an approach for systematic modeling and reasoning in conceptual design [11]. In [12] it has been extended to incorporate a visualization of geometric information and has introduced interval-temporal logics. It then becomes possible for the system architect to evaluate the consistency between spatial relations in a Function-Behaviour-State model and the corresponding geometric model.

Another design approach is represented by the Axiomatic Design, which is a general method for facilitating the synthesis of suitable design requirements, design solutions and design processes. Two principles must be verified according to Suh [13]: the independence axiom indicates to 'Maintain the independence of functional requirements', while the information axiom recommends to 'Minimize the information content of the design'. The independence axiom provides the designer with a measure for rating the correctness of the design, in insisting that an independent relationship, as represented by an uncoupled or decoupled design matrix, is essential for a successful design [14]. Evaluating design solutions according to these two axioms aims at verifying that the system is well designed but does not guarantee that the solutions are the most satisfying to fulfill the stakeholder's needs.

In order to qualify a mechatronic system, a mechatronic index is presented in [15] in terms of flexibility, intelligence and complexity, as these three characteristics account for much of mechatronic products. The intelligence level of a mechatronic system is determined by both its control functionality (which includes programmability, self-diagnosis, self-repair, negotiation, learning and self-organization) and its information computing ability (e.g. knowledge discovery and analysis, inference mechanism and communication) from the low-level control to the general management level. The flexibility property of a mechatronic system translates its capacity to easily 
change in order to fit new requirements or situations. Complexity is a consequence of the tradeoff involved when increasing the intelligence and flexibility and moreover may be observed through seven indices (including quantity of components, number of interconnections, number of design solution alternatives and number of feedback loops). The benefit of monitoring such indexes is to help mechatronic engineers in better designing their systems (i.e. a verification point of view) by taking into account the typical characteristics of mechatronic products: flexibility, intelligence, complexity, and strongly-coupled physical phenomena.

The approach of evaluation exposed in this paper is complementary to the above research works as it focuses on how to rank ASDS outputted from the synthesis activities and how to select the most satisfying for the stakeholders (i.e. more of a validation point of view than of a verification point of view). For this purpose, we adopt the Systems Engineering framework which guides the engineers throughout the macro-level design phases without imposing any specific method to synthesize solutions. The specificity of evaluating mechatronic design lies in taking into account, at the earliest possible stage in the design development cycle, the many relevant discipline requirements, in addition to the system architect requirement, in order to design a product.

\section{The evaluation process within the SE framework}

Systems Engineering (SE) is an interdisciplinary and collaborative approach based on the standards ISO/IEC 15288 [16], ANSI/EIA 632 and IEEE 1220, as well as on conceptual guidelines, e.g. SEBoK [17] and best practice guidelines, e.g. INCOSE handbook [18] or Nasa handbook [2]. SE promotes vocabulary, concepts and a comprehensive approach that facilitates communication within a design team, regardless of the underlying technological field, and moreover relies on a set of standardized processes and activities that have become widely and successfully applied across various industrial fields $[19,20,21]$.

Among the aforementioned components, five technical processes run in an iterative manner when engineering a system. These processes, identified in Fig. 2, are [17]: Mission analysis, Stakeholders' needs and requirements, System requirements, Logical architecture, and Physical architecture. They are supported by three services, namely: System verification, System validation, and System analysis.

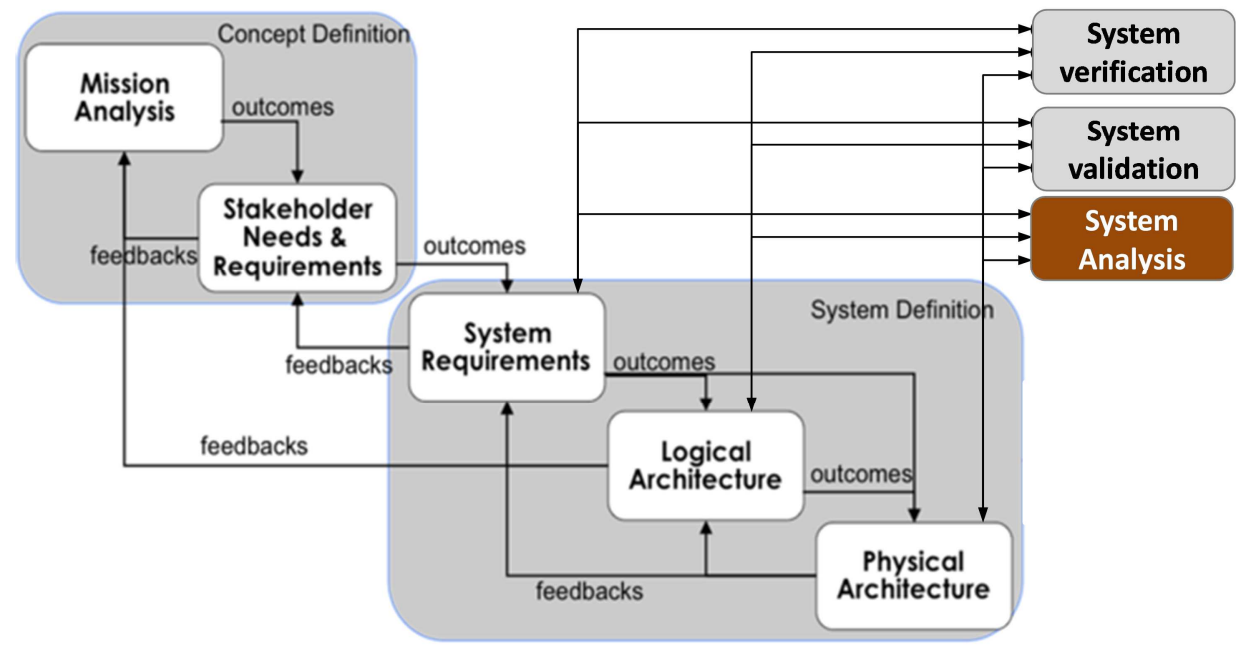

FIGURE 2: Technical and support processes in SE

Evaluation, is indeed an important activity of System Analysis in SE since according to standards (EIA 632, I'IEEE 1220, SEBok 2013) technical performance assessment and tradeoff studies are necessary activities of SE. 
Thus this paper addresses the set of System Analysis issues and focuses in particular on evaluating the product under design. The next section will present the generic activities associated with the evaluation process.

\subsection{Activities composing the evaluation process}

An evaluation is requested each time engineering choices must be made or justified, e.g. choice of major operations and solution concepts, research and resolution of inconsistencies between requirements, choice of logical vs. physical architecture. The criteria required to evaluate design progress actually evolve in the same manner as design model criteria [22].

This evaluation process includes generic activities, as summarized in Fig. 3. The main activities composing the evaluation process consist of: defining the evaluation objectives ("why" and "what"), preparing the job ("how"), producing and analyzing results ("do" and "check"), and finally delivering outcomes ("conclude"). Briefly summarized below are the individual tasks relevant to these successive stages.

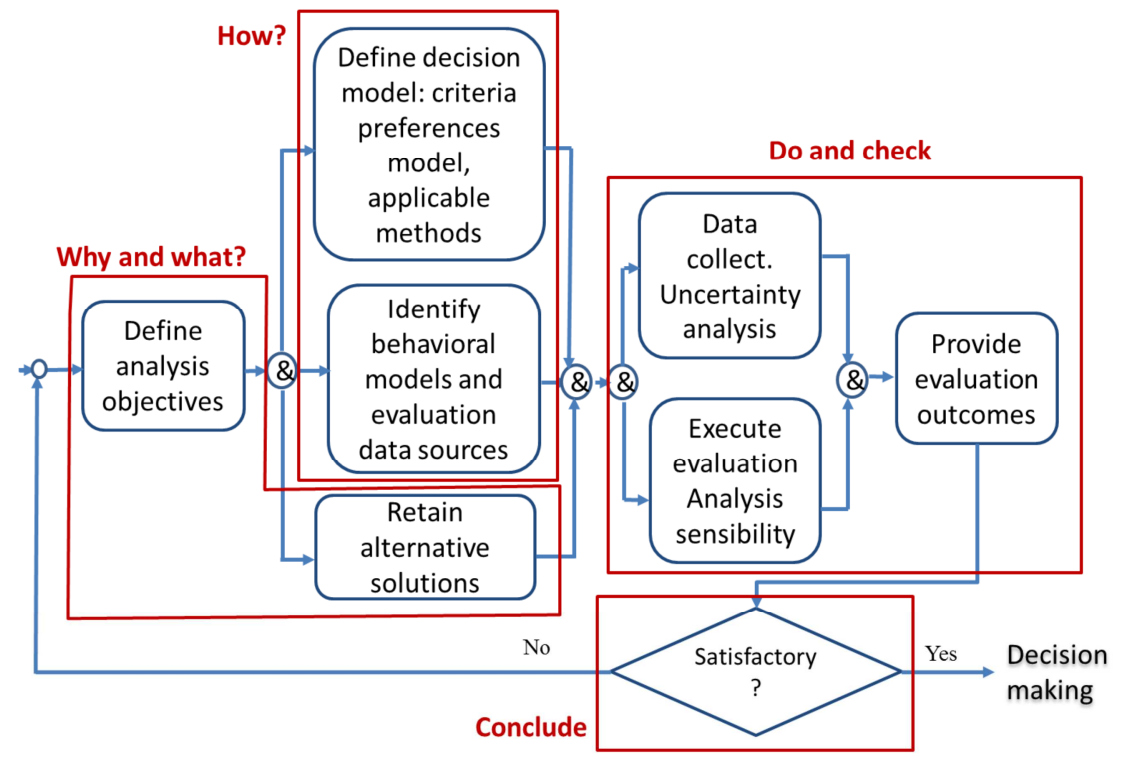

FIGURE 3: Flowchart of activities in the Evaluation Process

'Why and what' activities: After specifying the scope, objectives and means available to perform the requested evaluation, only the ASDS that satisfy the non-negotiable technical and economic criteria are to be selected for more in-depth analysis. It is generally recommended that the number of alternatives be limited (in the range of 3 to 7) and moreover that all candidate solution characteristics be sufficiently distinct.

'How' activities: Since the evaluation process constitutes the backbone of the decision-making process, the decision-making model must be specified. Such a model takes into account core elements, e.g. evaluation criteria, stakeholders' preferences, the scoring function used to assign desirability levels to system attributes, and the aggregation methods chosen to provide ASDS with the overall desirability levels used to classify or rank design solutions [15].

Regarding the evaluation of product effectiveness, three main families of multi-criteria decisionmaking methods can be distinguished [23]: total aggregation methods [24], paired comparison methods [25], and outranking approaches [26]. The aggregation methods most frequently used in design are based on Multi-Attribute Utility Theory according to which local 'utility functions' are defined so as to quantify on a standardized scale (e.g. [0 1]) stakeholders' preferences about the 
'attributes' of the designed artifact. These local utility values are then aggregated into an overall utility through application of a mathematical formalism (usually additive or multiplicative). The paired comparison methods (such as Analytical Hierarchical Process) are employed to both model stakeholders' preferences and rank alternatives. The primary outranking methods assume datasets similar to those assumed by the Multi-Attribute Utility Theory, yet allow defining just a partial ranking on the set of alternatives, which contains the most promising alternatives of the decisionmaking problem. In 'Electre III', an outranking degree is computed that reflects outranking credibility among several alternatives [23].

Since the characteristic values of ASDS are not directly measurable from the product, they need to be predicted from models (referred hereafter as behavior model). At the conceptual design stage, behavior models tend to be coarse whereas at the detailed design stage, more specialized and precise models must be taken into consideration in order to derive product feature data. Some activity within the evaluation process thus consists of identifying which level of modeling granularity is required and then ordering additional modeling and analysis work. More generally speaking, evaluation relies on relevant knowledge based on engineering rules, standards, expert reports, simulations and analysis reports from previous completed projects or from measurements carried out on prototypes. In any event, it is necessary to estimate the degree of uncertainty associated with collected data and perform the evaluation accordingly [27].

'Do and check' activities: ASDS are assessed or ranked according to the selected multi-criteria analysis method. Sensitivity analyses are also conducted to determine whether the resulting classification or ranking is sufficiently robust, e.g. if the classification/ranking remains unchanged in the event of small input data variations. If the evaluation objectives are not met or if results lack adequate differentiation or show excessive dependence on the evaluation methods, then the evaluation process should return to one of its previous steps.

'Conclude' activity: When the objectives are met, a report is produced and delivered to decisionmakers; this report includes: information on assessment objectives, the selected ASDS, the data sources along with their degrees of uncertainty, the decision-making model, evaluation results with their sensitivity analyses, and justifications of every choice made during the evaluation process.

The conceptual data model shown in Fig. 4 allows characterizing and describing the relevant concepts and relationships from the SE context that must be taken into consideration throughout the evaluation activities, from the conceptual design phase to the detailed design phase. As displayed in a UML class diagram, each class describes a requested concept from the domain and is characterized by a set of typical attributes and relations. Each relation in turn describes and formalizes a link (i.e. dependence relationship) between two concepts. As such, this conceptual data model corresponds to the abstract syntax, i.e. the vocabulary and basic grammar of the meta-model of the so-called 'evaluation language' proposed herein. Moreover, this language is indeed an extension or enrichment of Systems Engineering (SE) vocabulary and thus of the engineering language currently proposed in an MBSE context [28]. The section (2.2) presents the main classes of concepts and relations being considered among the three domains involved in the evaluation process: technical requirements, candidate solutions, and evaluations.

\subsection{A conceptual data model for evaluation data characterization within the SE framework}

According to Blanchard and Fabricky [8], the 'Design Consideration' concept designates any element of the complete set of attributes and characteristics potentially exhibited by: a system being engineered, a product, or a service. 'Design Considerations' are involved during the customer requirement analysis to facilitate the stakeholders' needs expression. They are also proved to be useful in analysis activities for the purpose of fully characterizing the product being designed and hence revealing the expected, as well as unexpected, behavior or properties. Among the set of 
'Design Considerations', let's distinguish the 'Design Dependent Parameters', which are controlled by the designers (e.g. geometric dimensions, weight, reliability, maintainability or other-ilities [29]). Each instance of 'Design Dependent Parameters' generates a design candidate solution. 'Input Design Dependent Parameters' (iDDPs) are 'Design Dependent Parameters' the values of which are chosen by the designers and determine other 'Design Dependent Parameters' values. The iDDPs which can be coded numerically referred to as design variables. Design variables include optimization variables used to improve a satisfactory solution even further. 'Output Design Dependent Parameters' (oDDPs) are 'Design Dependent Parameters' the values of which are generally computed from behavior models and iDDPs values. 'Output Design Dependent Parameters' are parameters of interest for the stakeholders and are used to evaluate an ASDS.

Energy autonomy is an example of 'Design consideration' for an electrical wheelchair. Then an oDDP can be the 'number of km travelled without reloading in energy' and an iDDP can be the 'battery capacity'.

The 'Design Independent Parameters' are known as "incurring" factors (for example: labor costs, variation of raw material characteristics, the market entry of a new competing product, energy cost, a new regulation etc.). 'Design Independent Parameters' are not controlled by the designers but they must be taken into account by decision-makers when selecting the most promising design solutions.

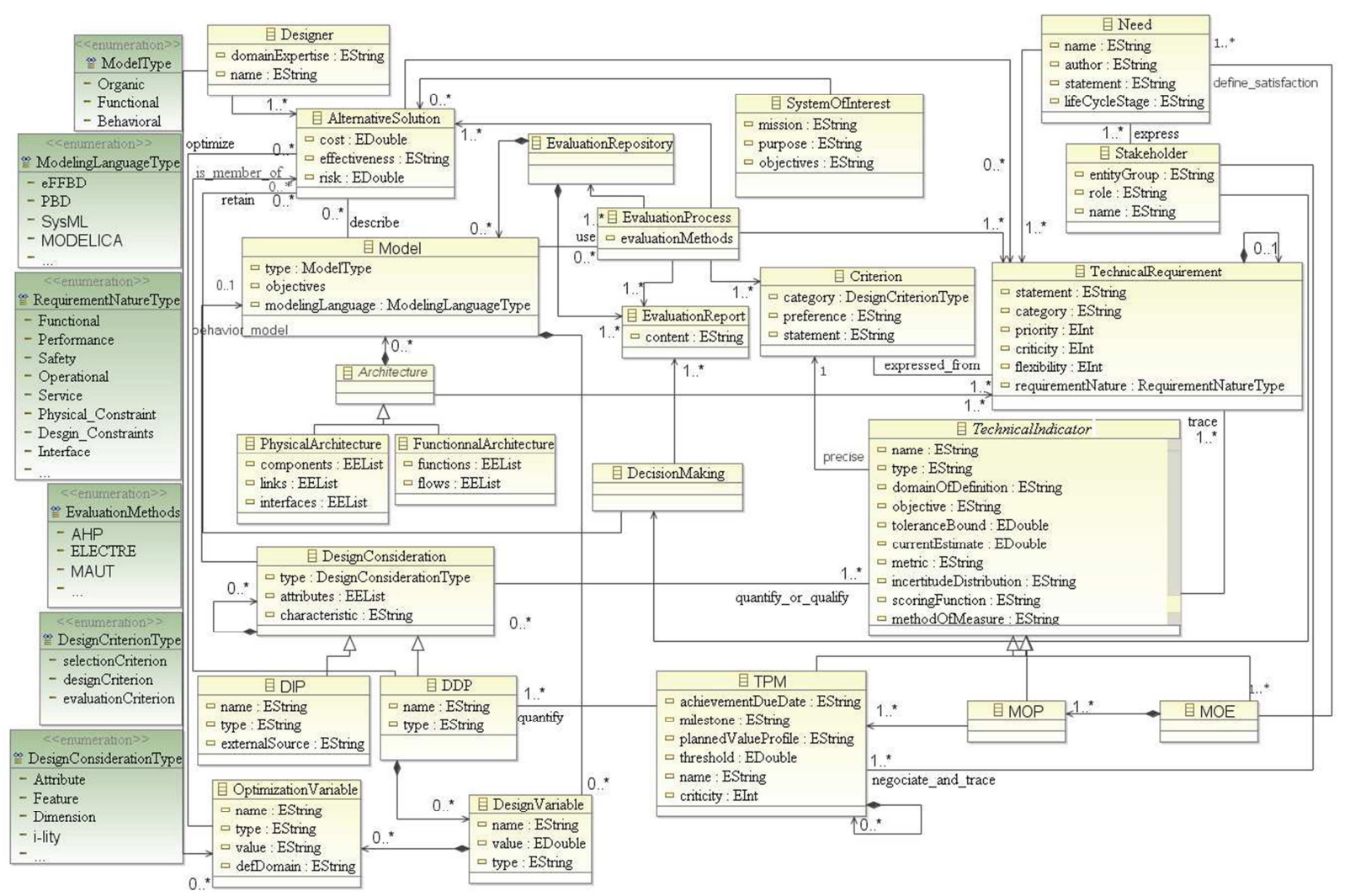

FIGURE 4: A Conceptual Data Model for characterizing evaluation data in an SE context

Considering multi-criteria decision-making methods (such as the ones cited in section 2.1 or the proposed one in section 4) the evaluation process establishes the level of adequacy between a consistent set of technical requirements (e.g. translations of stakeholders' needs by designers in considering the entire product life cycle) and ASDS. The evaluation criteria determine the principles or rules introduced to judge the level of adequacy. The evaluation criteria are specified by technical indicators (TIs), whose attributes comprise: the rating scales, objectives, target levels, and current level. A TI objective is specified by a single performance requirement or a single constraint. 
While defining needs, attention turns to identifying MOEs (Measures Of Effectiveness), which are mandatory TIs to ensure system success. Inability to reach an MOE objective is considered a cause of failure [2].

Not directly estimable during the design, MOEs can be decomposed into MOPs (Measures of Performance), which are TIs associated with the product's physical or functional properties. MOPs may in turn be broken down into 'Technical Performance Measures' (TPMs) or characteristics, which are measured from attributes inherent to the designed product [8]. Current TPM values are in fact derived from oDDP values. For instance let us define some MOE, MOP and TPM when designing an electrical assistance for a wheelchair. According to the stakeholders, the 'type of job that such assistance allows to practice in outside conditions' is a MOE (the MOE objective is to be able to work as a roundsman in a company). Such a MOE can be decomposed in several MOPs, the 'capacity to drive outside' is one of them (the MOP objective is to be able to drive on unsurfaced roads). The MOP can be decomposed in several TPMs, the 'capacity to climb sloping road' is one of them (the TPM objective is to be able to climb $10 \%$ slopes with a $120 \mathrm{~kg}$ total charge).

ASDS are described by their functional and physical architectures models (generated by SE activities) and also by behavior models built up to lead analysis studies and to calculate oDDP values. The knowledge elements needed to perform such analysis and the results of evaluation reports are collected in the 'Evaluation repository'.

In order to be able to analyze the impacts of design choices on criteria TI current values, it is first necessary to identify which TI current value (i.e. oDDP values) can be (adversely or positively) affected by iDDPs and then to determine the magnitude of such impacts. The next two sections are intended to propose some resolution to these issues conforming to the conceptual data model shown in Fig. 4.

\section{Identification of potential impacts}

In complex system design, it is not altogether obvious whether or not an iDDP exerts potential impact on an oDDP. The following section will analyze how traceability relations stemming from both SE models and the conceptual data model (Fig. 4) can actually help detect potential influence relations between DDPs.

Without imposing restrictive constraints on system architects, we are able to postulate that (Fig. 4):

- Any evaluation criterion is expressed using at least one technical requirement;

- Any criterion is specified by at least one technical indicator(TI);

- Any TI is associated with one and only one non-functional requirement (more precisely with one performance requirement or with one constraint).

Based on these assumptions and by utilizing the traceability relationships between SE elements in system models, the influence relations between TIs and iDDPs can be underscored so as to identify what might generate some impact. The formalization of such a notion will be provided hereafter.

\subsection{Traceability relations}

Traceability is defined as the ability to describe and track the life of a requirement from its origin (in the design phase) to deployment (in the operations phase) [30, 31]. The main needs behind introducing traceability in SE are summarized in Table 1. 


\begin{tabular}{|l|l|}
\hline \multicolumn{1}{|c|}{ Need } & \multicolumn{1}{|c|}{ Description } \\
\hline $\begin{array}{l}\text { Coverage } \\
\text { analysis }\end{array}$ & $\begin{array}{l}\text { Are all requests for features in the } \\
\text { delivered product? } \\
\text { Do test cases exist for all functional and } \\
\text { non-functional requirements? }\end{array}$ \\
\hline $\begin{array}{l}\text { Impact } \\
\text { analysis }\end{array}$ & $\begin{array}{l}\text { Tracing from the impacted features to } \\
\text { the actual system design to determine } \\
\text { the extent of eventual modifications }\end{array}$ \\
\hline $\begin{array}{l}\text { Derivation } \\
\text { analysis }\end{array}$ & $\begin{array}{l}\text { Help discover the origin and rationale of a } \\
\text { function; alternatively, a component } \\
\text { requirement is traced back to the original } \\
\text { rationale for its creation }\end{array}$ \\
\hline
\end{tabular}

TABLE 1: Traceability needs in an SE process

A more formal definition can be provided for:

- A 'traceability relation', denoted $\mathfrak{I}$ on $\mathrm{ExE}$, with $\mathrm{E}$ a set of artefacts, is a partial relation on ExE that is non-reflexive, non-symmetric and non-transitive. For example, the SE relation 'refines' (requirement $\mathrm{b}$ refines requirement $\mathrm{a}$ ) defines a 'traceability relation' on RxR where $R$ is the set of the artefacts 'system requirements'. SE relations have distinct semantics (refines, built from, specifies...); traceability relations have not.

- A 'traceability link', denoted $\tau$, is an oriented link between two artefacts; it is associated with a traceability relation between one source artefact ' $a$ ' and one target artefact ' $b$ ', such that $b$ $=\tau(a)$. So, if $\mathfrak{I}(a, b)$ is true then it exists $\tau$ such as $b=\tau(a)$. The set of artefacts connected by traceablity links forms a digraph.

- If $\mathfrak{I}_{1}$ and $\mathfrak{I}_{2}$ are two traceability relations defined on ExE, then $\mathfrak{I}_{1} 0 \mathfrak{I}_{2}$ is a traceability relation on ExE.

Any artifact a, with $a \in E$, can display an impact $\delta_{\text {ba }}$ on other artefacts $b$ of $E$ if and only if a is the source of at least one traceability link $\tau$ with $b=\tau(a)$. The set of artefacts that are in relations by $k$ successive compositions of a traceability relation $\mathfrak{I}$ is thus expressed as:

$I^{k}(a)=\left\{b \mid b=\tau^{k}(a)\right\}$, where $\tau^{k}(a)$ is $\tau\left(\tau^{k-1}(a)\right)$; the coverage of element a is: $I_{C}(a)=\bigcup_{q=1}^{k} I^{q}(a)$

Such formalization is used to compute the set of artefacts that can potentially impact or be impacted by others as proposed in next section.

\subsection{Using traceability relations to identify potential impacts}

Table 2 presents examples of relationships between entities according to an example of an SE metamodel (i.e. a model proposed by one of the MBSE solution providers; other meta-models derived from other providers may also be used)[32]. To these SE relationships, are associated 'traceability links' as defined above.

According to the conceptual data model shown in Fig. 4, we propose adding the relationships between criteria, requirements and Technical Indicators (TIS) to the basic relationships listed in Table 2, with the outcome shown in Table 3. 


\begin{tabular}{|c|c|c|}
\hline Source / Target & $\begin{array}{l}\text { Target / } \\
\text { Source }\end{array}$ & $\begin{array}{l}\text { SE relations and their associated } \\
\text { (traceability links) }\end{array}$ \\
\hline Functional requirement & Function & Basis of $\left(\tau_{b_{-} o f}\right) /$ Based on $\left(\tau_{b_{-} o n}\right)$ \\
\hline Performance requirement & Function & Specifies $\left(\tau_{s}\right) /$ Is specified by $\left(\tau_{s_{-} b y}\right)$ \\
\hline $\begin{array}{l}\text { Performance requirement, } \\
\text { Constraint }\end{array}$ & Component & Specifies $\left(\tau_{s}\right) /$ Is specified by $\left(\tau_{s_{-} b y}\right)$ \\
\hline Function & Component & Is allocated to $\left(\tau_{a_{\_} t o}\right) /$ Performs $\left(\tau_{p}\right)$ \\
\hline Function & Function & Decomposes $\left(\tau_{d}\right) /$ Decomposed by $\left(\tau_{d \_b y}\right)$ \\
\hline Component & Component & Built from $\left(\tau_{b_{-} \text {from }}\right)$ / Built in $\left(\tau_{b_{-} i n}\right)$ \\
\hline Requirement & Requirement & Refines $\left(\tau_{r}\right) /$ Is refined by $\left(\tau_{r_{-} b y}\right)$ \\
\hline Requirement & Interface & Specifies $\left(\tau_{s}\right) /$ Is specified by $\left(\tau_{s_{b} b y}\right)$ \\
\hline Interface & Component & Joins $\left(\tau_{j}\right) /$ Joined to $\left(\tau_{j_{-} t o}\right)$ \\
\hline Interface & Link & Comprised of $\left(\tau_{c m}\right) /$ Comprises $\left(\tau_{c m \_b y}\right)$ \\
\hline Link & Item & Transfers $\left(\tau_{t}\right) /$ Is transferred by $\left(\tau_{t_{-b y}}\right)$ \\
\hline
\end{tabular}

TABLE 2: Traceability relations between SE entities [23]

One criterion and one TI may be correlated with the same requirement if and only if this requirement specifies the $\mathrm{Tl}$ objective in its formulation. The inability to link any of the Tls with a single performance requirement or with a single constraints attests to a certain level of incompleteness in the set of requirements.

\begin{tabular}{|l|l|l|}
\hline \multicolumn{1}{|c|}{ Source /Target } & \multicolumn{1}{c|}{$\begin{array}{c}\text { Target } / \\
\text { Source }\end{array}$} & \multicolumn{1}{c|}{$\begin{array}{c}\text { SE relations and their associated } \\
\text { traceability links }\end{array}$} \\
\hline Criterion & Criterion & Decomposes $\left(\tau_{d c}\right) /$ Decomposed by $\left(\tau_{d c_{-} b y}\right)$ \\
\hline $\mathrm{TI}$ & Criterion & Precises $\left(\tau_{p r}\right) /$ Is precised by $\left(\tau_{p r_{-} b y}\right)$ \\
\hline Requirement & Criterion & Expresses $\left(\tau_{\text {ex }}\right) /$ Expressed from $\left(\tau_{e_{-} \text {from }}\right)$ \\
\hline $\mathrm{TI}$ & $\begin{array}{l}\text { non-funct. } \\
\text { requirement }\end{array}$ & Traces $\left(\tau_{t r}\right) /$ Is traced by $\left(\tau_{\text {tr_by }}\right)$ \\
\hline
\end{tabular}

TABLE 3: Extension of traceability relation between SE entities

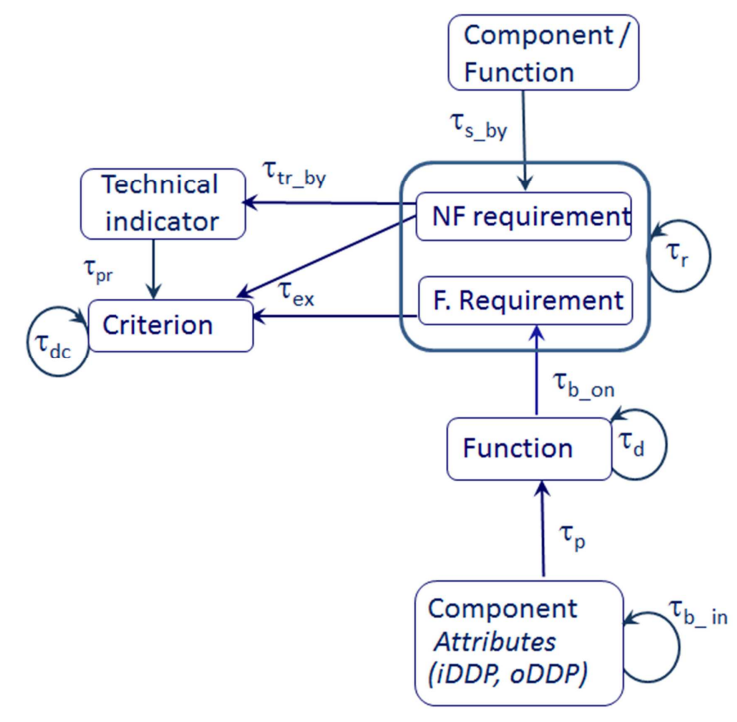

FIGURE 5: Identifying potential impact from traceability links

Using compositions of the traceability links presented in Tables 2 and 3 can serve to highlight potential impacts between any function parameter (an instance of an iDDP) or any component 
attribute (another instance of an iDDP) and the evaluation criteria and their associated Tls (the current value of an $\mathrm{TI}$ is an instance of an oDDP).

Thus, if the value of some iDDP (attribute of a component) is changed, traceability links $\tau_{b_{-} i n}, \tau_{p,} \tau_{d}, \tau_{b_{-} o n}, \tau_{r}, \tau_{e x}$ spread the possibility of impact to the evaluation criteria (Fig. 5). Similarly, from traceability links, $\tau_{p r_{-b y}}, \tau_{t r_{-b y}}, \tau_{s_{-} b y}, \tau_{r_{-} b y}, \tau_{a_{-t o}}$ it is possible to identify the functions and components potentially impacted by a change in some TI objective value of one criterion.

The TI value reached by an ASDS is derived from an oDDP value. According to available knowledge, the transformation between iDDPs and oDDPs may be either a quantitative model with a low level of uncertainty or a qualitative one with higher uncertainty.

The latter case is critical during product design since it occurs at the very earliest stage of the design development cycle, when both the future success and cost issues of the design project are being determined in full. For this reason, Section 4 will focus on this SE analysis, which often remains inadequate in its methodology.

\section{Decision-making aid in designing complex products in an SE context}

\subsection{Linking a predictive system model with a decision-making model}

A number of notations will be introduced in this subsection.

Let's start by considering a situation with $m$ iDDPs. Each iDDP is assigned a value from the set denoted $X_{j}$, with $j=1, \ldots, m$, where $X=X_{1} \times X_{2} \times \ldots \times X_{m}$ denotes the set of all m_tuple $x=\left(x_{1}, x_{2}, \ldots, x_{m}\right)$ and where $x_{j} \in X_{j}$ is the value of the $j^{\text {th }}$ iDDP. $X_{j}$ may be a set of nominal, discrete or continuous values. Each element of $X$ represents an ASDS.

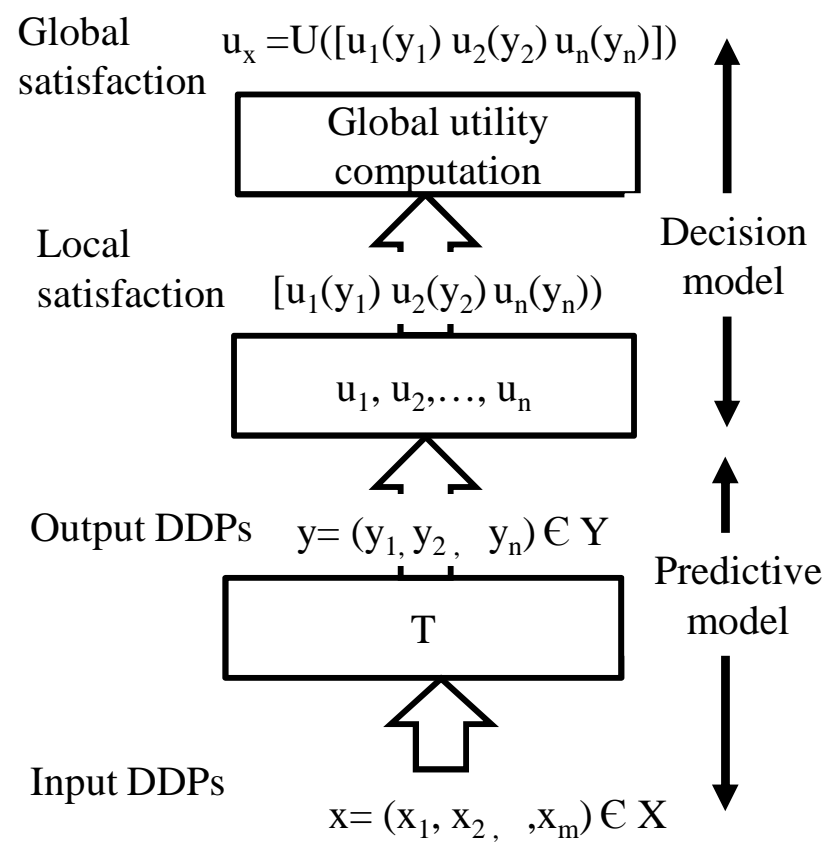

FIGURE 6: Merging a system predictive model with a decision-making model

For instance let's consider the design of a motor-wheel for a wheelchair: the diameter of the wheel in inches, the nominal torque provided by the electrical engine in N.m, the reduction ratio of the 
gearbox are examples of iDDPs. The iDDP $\mathrm{j}$ 'nominal torque of the electrical engine' may take its values $x_{j}$ in the interval $X_{j}=[100,600] \mathrm{mNm}$.

Let's also consider that the system may be described by $\mathrm{n}$ oDDPs relative to the decision-making criteria. Each oDDP is assigned values from the set denoted $Y_{i}$, with $i=1, \ldots, n$ and with $Y=Y_{1} \times Y_{2} \times \ldots \times Y_{n}$ denoting the set of all n_tuple $y=\left(y_{1}, y_{2}, \ldots, y_{n}\right)$, where $y_{i} \in Y_{i}$ is the value assigned to the $\mathrm{i}^{\text {th }}$ oDDP. $Y_{i}$ may be a set of nominal, discrete or continuous values. For instance the oDDP i 'Current consumption' may take its values $y_{i}$, in the interval $Y_{i}=[1,5] \mathrm{A}$.

Each input configuration in $X$ corresponds to an output in $Y$ representing the system characteristics of interest. Let's indicate this correspondence by the transformation: $T=\left(T_{1}, \ldots, T_{n}\right)$, where: $T_{i}: X \rightarrow Y_{i}, i=1, \ldots, n$ (Fig.6).

T may be a formal transformation deduced either from physical laws or from validated knowledge in the field. For example, a dynamical model of the wheelchair can compute the 'Current consumption' from the values of iDDPs such as 'nominal torque of the engine', 'diameter of the wheel', 'reduction ratio of the gearbox' and others impacting iDDPs. In general however, $\mathrm{T}$ is not explicitly known and, in the most favorable case, it can be approximated by a predictive model [33].

The oDDPs must then be interpreted in terms of satisfaction with respect to the stakeholders' goals. The Multi-Attribute Utility Theory allows expressing the degree of satisfaction of an oDDP by a realvalued utility function in $[0,1][34,35,36]$. Let's denote therefore for $i \in\{1, \ldots, n\}, u_{i}$ ( $\left.u_{i}: Y_{i} \rightarrow[0,1]\right)$ the real-valued utility function for oDDP $i$. For $y_{i} \in Y_{i}, u_{i}\left(y_{i}\right)$ reflects the extent to which the goal associated with the TPM $i$ is satisfied by the value $y_{i}$.

Then the satisfaction level of a higher-level TI, in the hierarchy of criteria, e.g. a MOP or a MOE, in the decomposition tree containing TPM, should be computed using some aggregation operator U (Fig. 6). According to the Multi-Attribute Utility Theory, the weighted arithmetic mean can be the operator used to compute the global utility from the local utilities $u_{i}\left(y_{i}\right)$.

As noted above, transformation $\mathrm{T}$ is typically unknown or marred by uncertainty, and this mainly arises during the preliminary (or conceptual) design phase. The scenario is then often played out where experts or senior engineers are asked to advise on ASDS and judge the qualitative effect of design choices on stakeholder satisfaction. To properly treat this kind of model, we have relied on the research work presented in [37] regarding qualitative analyses.

\subsection{Qualitative analyses}

The notion behind the qualitative model in [37] is that an expert can express the impact of an iDDP instance directly as a degree of satisfaction of an oDDP. Our attention here is thus focused on the transformation $\mathrm{u}_{i} \mathrm{o} T_{i}, i=1, \ldots n$, which is approximated by a qualitative model (Fig. 7). Figure 7 has to be generated by the designer team in agreement with the stakeholders. Two scales are used in this context: a positive ordinal scale $P S$ and a negative ordinal scale $N S$. If the impact of a given instance $x_{j}$ of iDDP $X_{j}$ on the $i t h$ oDDP is positive, its values are taken from $P S$ (ex: strong, medium, low, etc.) and the notation $\delta_{i j}^{+}$is introduced. On the other hand, if the impact is negative, its values are derived from $N S$ (ex: strong, medium, low, etc.) with use of the notation $\delta_{i j}^{-}$. Should the expert not be able to decide, the unknown impact is denoted $\delta_{i j}^{u}$. This paper has assumed that $P S=N S$ and 
an absence of impact is represented by the value 0 (which corresponds to the lower value on the $P S$ scale).

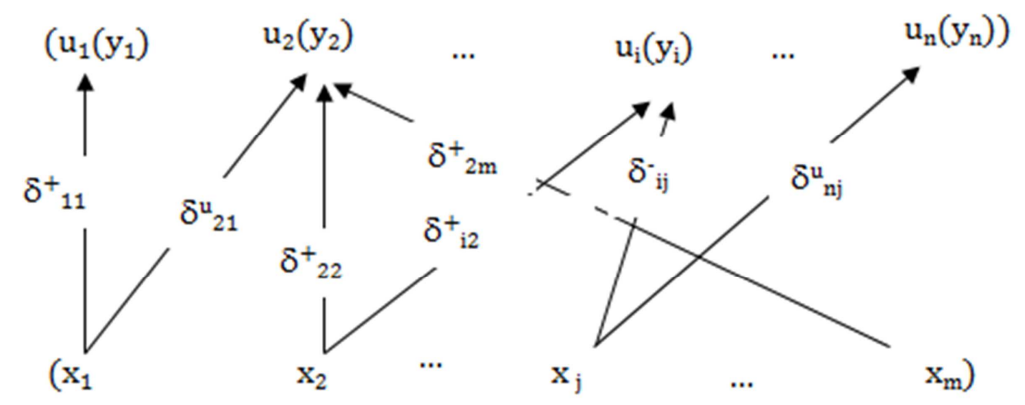

FIGURE 7: Influence digraph ( $\delta^{+}$: improvement, $\delta$ : degradation, $\delta^{u}$ : unknown)

Let's now introduce two sets: $S_{i, x}^{+}=\left\{j \in\{1, \ldots, m\}: x_{j}\right.$ impacts $i$ positively $\}$ (i.e. for each m_tuple $x$ the set of iDDP $j$ that exerts a positive impact on oDDP $i$ ), and $S_{i, x}^{-}=\left\{j \in\{1, \ldots, m\}: x_{j}\right.$ impacts $i$ negatively $\}$ (for each $\mathrm{m}_{-}$tuple $x$ the the set of iDDP $\mathrm{j}$ that negatively affects oDDP $i$ ).

To assign the degree of impact of an ASDS $x=\left(x_{1}, \ldots, x_{m}\right) \in X$ on an oDDPi, [36] introduced two aggregation operators along with two propagation constraints. Each combination of an aggregation operator with a propagation constraint yields a decision-maker's attitude. The following pessimistic or optimistic attitude of the decision-maker can serve as a guide. Indeed, the estimation of the ASDS $\left(x_{1}, \ldots, x_{m}\right)$ merged impact on oDDPi naturally depends on system behavior, as well as on the decision maker's decisional behavior: a pessimistic attitude (whereby a risk aversion position will lead to concluding the most highly negative merged impacts) vs. an optimistic attitude (whereby risk acceptance will conclude the most highly positive merged impacts).

Let's denote $\delta_{i, x}$ the impact of ASDS $x$ on oDDP $i$. We also consider that $\delta_{i, x} \in P S \times N S$ and can be represented by the pair $\left(\delta_{i, x}^{+, a t}, \delta_{i, x}^{-, a t}\right)$, as proposed [38], where $\delta_{i, x}^{+, a t}$ (resp. $\delta_{i, x}^{-, a t}$ ) corresponds to an aggregation of positive impacts (resp. negative impacts) and at $\in\{1,2\}$ corresponds to the decisionmaker's attitude (" 1 " encodes the pessimistic attitude and " 2 " the optimistic attitude). In the following, $\left(\delta_{i, x}^{+, a t}, \delta_{i, x}^{-, a t}\right)$ will be explained for both these attitudes.

The pessimistic attitude (at=1 in $\delta_{i, x}^{+, a t}$ and $\delta_{i, x}^{-, a t}$ ) is modeled by assigning the least positive impact as the aggregation of positive impacts (less favorable case) and the worst negative impact as the aggregation of negative impacts (less favorable case again), in which case the propagation constraint consists of comparing the least positive impact value with the worst negative impact value on oDDP $\mathrm{i}$, resulting in:

$$
\delta_{i, x}^{+, 1}=\left\{\begin{array}{c}
\min _{j \in S_{i, x}^{+}} \delta_{i j}^{+} \text {if } \min _{j \in S_{i, x}^{+}} \delta_{i j}^{+}>\max _{j \in S_{i, x}^{-}} \delta_{i j}^{-} \\
0 \text { otherwise }
\end{array} \quad \delta_{i, x}^{-, 1}=\left\{\begin{array}{c}
0 \text { if } \min _{j \in S_{i, x}^{+}} \delta_{i j}^{+}>\max _{j \in S_{i, x}^{-}} \delta_{i j}^{-} \\
\max _{j \in S_{i, x}^{-}} \delta_{i j}^{-} \text {otherwise }
\end{array}\right.\right.
$$


In this case, an unknown impact corresponds to a negative impact and is equal to the top of the $N S$ scale.

The optimistic attitude (at $=2$ in $\delta_{i, x}^{+, a t}$ and $\delta_{i, x}^{-, a t}$ ) is modeled by assigning the highest positive impact as the aggregation of positive impacts and the worst negative impact as the aggregation of negative impacts. In this case, the propagation constraint consists of comparing the highest positive impact value with the worst negative impact value on oDDP $i$, hence:

$$
\delta_{i, x}^{+, 2}=\left\{\begin{array}{c}
\max _{j \in S_{i, x}^{+}} \delta_{i j}^{+} \quad \text { if } \max _{j \in S_{i, x}^{+}} \delta_{i j}^{+}>\max _{j \in S_{i, x}^{-}} \delta_{i j}^{-} \\
0 \text { otherwise }
\end{array} \quad \delta_{i, x}^{-, 2}=\left\{\begin{array}{c}
0 \text { if } \max _{j \in S_{i, x}^{+}} \delta_{i j}^{+}>\max _{j \in S_{i, x}^{-}} \delta_{i j}^{-} \\
\max _{j \in S_{i, x}^{-}} \delta_{i j}^{-} \text {otherwise }
\end{array}\right.\right.
$$

For this latter case, an unknown impact corresponds to an absence of impact and is equal to the bottom of the $P S$ scale.

Let's denote $\delta_{x}^{a t}=\left(\delta_{x}^{+, a t}, \delta_{x}^{-, a t}\right)$ as the impact of the m_tuple $x$ of iDDP on the $\mathrm{n}$ oDDPs. Then, in order to avoid risky choices, it can be reasonably stated:

$$
\delta_{x}^{a t}=\left(\delta_{x}^{+, a t}, \delta_{x}^{-, a t}\right)=\left(\min _{i}\left(\delta_{i, x}^{+, a t}\right), \max _{i}\left(\delta_{i, x}^{-, a t}\right)\right)
$$

Note that veto and dictator effects of some criteria through min and max operators can be smoothed when a weights distribution is assigned to the oDDPs as suggested in [39].

According to expressions (1) and (2), one of the values $\delta_{x}^{+, a t}$ or $\delta_{x}^{-, a t}$ is equal to zero.

The most promising ASDS would then be the one with the highest positive impact or otherwise the lowest negative impact on the $\mathrm{n}$ oDDP:

The most promising ASDS: $\left\{\begin{array}{l}\underset{x \in A S D S}{\arg \max } \delta_{x}^{+, a t} \text { if } \exists i: \delta_{i, x}^{+, a t} \neq 0 \\ \underset{x \in A S D S}{\arg \min } \delta_{x}^{-, a t} \text { otherwise }\end{array}\right.$

Section 5 will illustrate this type of qualitative analysis through designing an electrically-assisted device for a wheelchair.

\section{Illustrations}

This section will illustrate the proposed approach in the context of designing of a power-assisted wheelchair. Such an assisted wheelchair has been developed by a pluridisciplinary team on the mechatronics platform of Ecole des Mines d'Ales. The purpose of this electrical assistance is to promote employment opportunities for the mobility impaired. The system is being made available firstly to allow a person with reduced mobility to perform an outdoor job without undue fatigue and secondly to enable driving the wheelchair as if it were a manual device. The objectives adopted refer to the capability of moving on uneven ground and under exterior conditions. Constraints regarding maximum allowable speed, maximum dimensions and safety have been defined in the relevant set of regulations [40].

Once stakeholders' needs have been translated into a set of technical requirements (which should subsequently be enhanced as the design process progresses), the main principles of electrical assistance are to be analyzed and a concept of electrical assistance selected [41]. The selected product concept chosen in [41] is the concept of motor-wheel consisting in measuring the pushing 
force of the mobility-impaired individual on the handrail and then delivering additional torque on each wheel in proportion to the measured force.

During the preliminary design stage, a set of evaluation criteria and associated TIs are defined in accordance with the technical requirements; these elements are partially displayed in Fig. 8 . The mission of the assistance system is to help reduced mobility persons to be maintained in their job or to access to a new one. It is the real motivation of the customers (big firms which have obligation to employ reduced mobility persons). So the assistance system has to improve such persons mobility (needed, for instance, to practice a roundsman profession) but it must be quite easy and intuitive to use (as a manual wheel chair) in order the person to be able to do the job efficiently. So 'Employability' criterion must be satisfied to ensure success of this assistance system. As per the agreement with clients, this criterion is associated with the MOE 'Ability to perform a roundsman's job'. Such an 'Employability' criterion has been broken down into both a 'Mobility criterion' (with its associated MOP: 'Outside drive possibility') and a 'User Friendliness' criterion (with its MOP: 'Just an assistance').

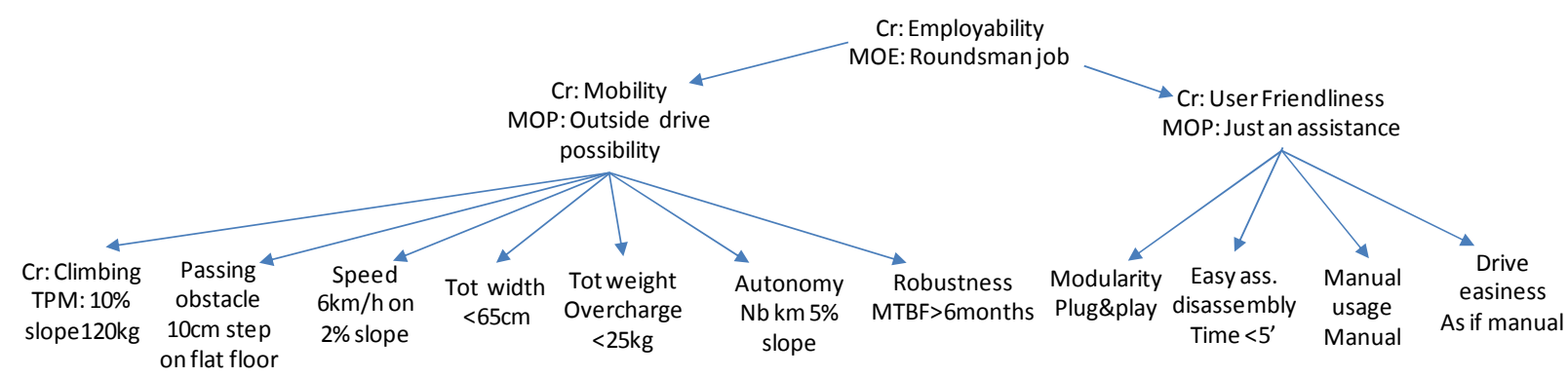

FIGURE 8: Decomposition of Criterion ( $\mathrm{Cr}$ ) and associated TIs (MOE,MOPs,TPMs)

Another lower level of the criterion hierarchy with associated TPM indicators is correlated with a set of more elementary oDDPs (such as the ability to: climb, overcome an obstacle, maintain average speed etc. as shown in Table 4). Let us remark that such a criteria hierarchy is not unique and may be different depending on the design team, but each criterion is expressed from technical requirements (according to the conceptual data model in Fig.4).

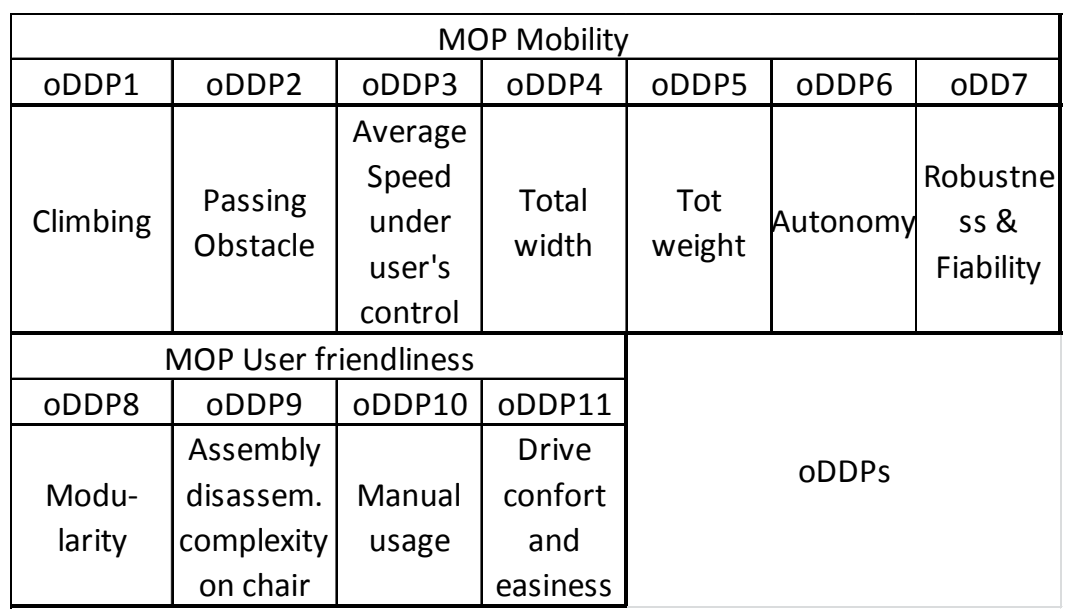

TABLE 4: output DDPs $\mathrm{i}, \mathrm{i} \in\{1, . ., 7\}$ relative to 'Mobility' and output DDPs $\mathrm{i}, \mathrm{i} \in\{8, . ., 11\}$ relative to 'User friendliness'

As the design progresses, these chief concerns must be handled, namely choices of: mechanical transmission and reduction (gears vs. rack and pinion), number of electric motors (one or more per wheel), location of the battery and electronics board (on the wheel vs. the chair), control type (reflex 
vs. smart), tuning facilities (with or without), and system status feedback to the user (rich or poor) as seen in Table 5. The iDDPs of table 5 have been identified by the designers within the development team. They result from the SE activities performed by the team and depend also upon the designers' experience. The example has been adapted for the sake of explanation but we have done the effort to stick the reality as well as possible. We do not focus in the paper on how ASDS are generated by the designers but on how choices can be made rationally between them within a multidisciplinary context. According to the identified iDDPs, the whole proposed method is all about to selecting some solutions from all possible combinations. As there is no incompatibility between iDDPs, the number of possible ASDS is $3^{2} \times 2^{6}=576$. The question is which ASDS are the most promising relatively to the main criteria: mobility, user friendliness and employability?

\begin{tabular}{|c|c|c|c|c|c|c|c|c|c|}
\hline \multicolumn{3}{|c|}{$\begin{array}{c}\text { iDDP1 } \\
\text { Reduction type }\end{array}$} & \multicolumn{3}{|c|}{$\begin{array}{l}\text { iDDP2 } \\
\text { Motors }\end{array}$} & \multicolumn{2}{|c|}{$\begin{array}{c}\text { iDDP3 } \\
\text { Battery location }\end{array}$} & \multicolumn{2}{|c|}{$\begin{array}{c}\text { iDDP4 } \\
\text { Wheel structure } \\
\text { rigidity }\end{array}$} \\
\hline $\begin{array}{l}\text { gear on } \\
\text { motor } \\
(1)\end{array}$ & $\begin{array}{l}\text { rack and } \\
\text { pinion } \\
(2)\end{array}$ & $\begin{array}{l}\text { mixed } \\
(3)\end{array}$ & $\begin{array}{c}1 \text { motor } \\
(1)\end{array}$ & $\begin{array}{c}3 \text { motors } \\
(2)\end{array}$ & $\begin{array}{c}>3 \\
\text { motors } \\
(3)\end{array}$ & $\begin{array}{c}\text { battery } \\
\text { location } \\
(1)\end{array}$ & $\begin{array}{c}\text { package } \\
\text { on chair } \\
(2)\end{array}$ & $\begin{array}{l}\text { high } \\
(1)\end{array}$ & $\begin{array}{c}\text { some } \\
\text { flexibiity } \\
(2)\end{array}$ \\
\hline \multicolumn{2}{|c|}{$\begin{array}{c}\text { iDDP5 } \\
\text { Electronics board } \\
\text { location }\end{array}$} & \multicolumn{2}{|c|}{$\begin{array}{c}\text { iDDP6 } \\
\text { Control type }\end{array}$} & \multicolumn{2}{|c|}{$\begin{array}{l}\text { iDDP7 } \\
\text { Setting }\end{array}$} & \multicolumn{2}{|c|}{$\begin{array}{c}\text { iDDP8 } \\
\text { Feedback to user }\end{array}$} & \multirow{2}{*}{\multicolumn{2}{|c|}{ iDDPs }} \\
\hline $\begin{array}{l}\text { on board } \\
\text { (1) }\end{array}$ & $\begin{array}{c}\text { on wheel } \\
\text { (2) }\end{array}$ & $\begin{array}{l}\text { smart } \\
\text { control } \\
(1)\end{array}$ & $\begin{array}{c}\text { reflex } \\
\text { control } \\
(2)\end{array}$ & $\begin{array}{c}\text { no tuning } \\
\text { possibilty } \\
\text { (1) }\end{array}$ & $\begin{array}{c}\text { tuning } \\
\text { posibility } \\
\text { (2) }\end{array}$ & $\begin{array}{c}\text { no } \\
\text { feedback } \\
\text { (1) }\end{array}$ & $\begin{array}{c}\text { feedback } \\
\text { to user } \\
(2)\end{array}$ & & \\
\hline
\end{tabular}

TABLE 5: input DDPs j, $\mathrm{j} \in\{1, \ldots 8)$

\begin{tabular}{|c|c|c|c|c|c|c|c|c|c|c|c|c|}
\hline & & \multicolumn{7}{|c|}{ MOP Mobility } & \multicolumn{4}{|c|}{ MOP User friendliness } \\
\hline & & oDDP1 & oDDP2 & oDDP3 & oDDP4 & oDDP5 & oDDP6 & oDDP7 & oDDP8 & oDDP9 & oDDP10 & oDDP11 \\
\hline \multirow{3}{*}{ iDDP1 } & $(1)$ & $+M$ & $+M$ & $+M$ & $-L$ & $-L$ & $-M$ & $-L$ & & & $-H$ & $-H$ \\
\hline & $(2)$ & $+\mathrm{L}$ & $+\mathrm{L}$ & $+M$ & $+\mathrm{L}$ & $+\mathrm{L}$ & $-L$ & $-\mathrm{H}$ & & & $-\mathrm{L}$ & $-\mathrm{L}$ \\
\hline & (3) & $+M$ & $+M$ & $+M$ & $\mathrm{~N}$ & $N$ & $-L$ & $-M$ & & & $-M$ & $-M$ \\
\hline \multirow{3}{*}{ iDDP2 } & (1) & & & & $-\mathrm{L}$ & $\mathbf{N}$ & $\mathbf{N}$ & $+M$ & $\mathrm{~N}$ & & $-H$ & U1 \\
\hline & $(2)$ & & & & $\mathrm{N}$ & $+\mathrm{L}$ & $-L$ & $+\mathrm{H}$ & $-L$ & & $-\mathrm{L}$ & U2 \\
\hline & (3) & & & & $N$ & $+\mathrm{L}$ & $-M$ & $-L$ & $-M$ & & $-M$ & U3 \\
\hline \multirow{2}{*}{ iDDP3 } & $(1)$ & & & & $+\mathrm{L}$ & & & $+\mathrm{L}$ & $+\mathrm{L}$ & $-M$ & $-M$ & $+\mathrm{L}$ \\
\hline & $(2)$ & & & & $\mathbf{N}$ & & $+\mathrm{L}$ & $+\mathrm{H}$ & $+\mathrm{H}$ & $-L$ & $-L$ & $+\mathrm{M}$ \\
\hline \multirow{2}{*}{ iDDP4 } & $(1)$ & & $+\mathrm{L}$ & & $\mathbf{N}$ & $-\mathrm{L}$ & $+M$ & $+\mathrm{H}$ & $-L$ & $-L$ & $-\mathrm{H}$ & $-\mathrm{H}$ \\
\hline & $(2)$ & & $+M$ & & $+\mathrm{L}$ & $+\mathrm{L}$ & $+\mathrm{L}$ & $+\mathrm{L}$ & $\mathbf{N}$ & $\mathbf{N}$ & $-\mathrm{L}$ & $-L$ \\
\hline \multirow{2}{*}{ iDDP5 } & $(1)$ & & $+M$ & & $+\mathrm{L}$ & & $+M$ & $+M$ & $+\mathrm{L}$ & $-\mathrm{H}$ & $-M$ & $+M$ \\
\hline & (2) & & $+\mathrm{L}$ & & $\mathbf{N}$ & & $+\mathrm{L}$ & $+\mathrm{L}$ & $+\mathrm{M}$ & $-L$ & $-L$ & $+\mathrm{L}$ \\
\hline \multirow{2}{*}{ iDDP6 } & (1) & & $+\mathrm{M}$ & $+\mathrm{L}$ & & & $+\mathrm{H}$ & & & & & $+\mathrm{H}$ \\
\hline & (2) & & $+\mathrm{L}$ & $-L$ & & & $+\mathrm{L}$ & & & & & $-L$ \\
\hline \multirow{2}{*}{ iDDP7 } & (1) & & & $-L$ & & & $\mathrm{~N}$ & $\mathrm{~N}$ & $+\mathrm{L}$ & $\mathrm{N}$ & $\mathrm{N}$ & $\mathrm{N}$ \\
\hline & (2) & $+M$ & $+M$ & $+\mathrm{L}$ & & & $+M$ & $+\mathrm{L}$ & $\mathbf{N}$ & $+\mathrm{L}$ & $-L$ & $+\mathrm{L}$ \\
\hline \multirow{2}{*}{ iDDP8 } & (1) & & & $-M$ & & & $\mathbf{N}$ & $\mathrm{N}$ & $+\mathrm{L}$ & $\mathrm{N}$ & $\mathrm{N}$ & $\mathrm{N}$ \\
\hline & (2) & & & $+\mathrm{L}$ & & & $+M$ & $+M$ & $\mathrm{~N}$ & $+\mathrm{L}$ & $-L$ & $+\mathrm{L}$ \\
\hline
\end{tabular}

TABLE 6: Example of impacts of instances of input DDPs $x j$ on output oDDP $i$ ( $\mathrm{H}$ : High, M: Medium, L: Low, N: null (0), $\mathrm{U}_{\mathrm{k}}$ : unknown)

No behavior model being available at this stage of the design process to compute how iDDPs values impact the oDDps the analysis relies on the expertise of a senior designer who provided advices relative to the impacts of such choices on the degree of utility of elementary oDDPs, as summarized in Table 6. The ordinal scale on which the impacts are assigned their values is $P S=N S=\{H, M, L, N, U\}$, 
where $\mathrm{N}$ denotes a zero impact $(0), \mathrm{L}$ denotes low impact, $\mathrm{M}$ denotes medium impact, $\mathrm{H}$ denotes high impact and $U$ an unknown impact (i.e. an impact is present, yet its strength raises doubts).

For instance, equipping the motor wheel with one $\mathrm{CC}$ motor, the impact on the 'total wheelchair width' (oDDP4) satisfaction level is (-L) due to the motor large diameter while it is $(+L)$ if equipped with smaller diameter motors. 'Tuning facility' increases satisfaction level for 'climbing capacity' (+M) or 'overcoming obstacles capacity' (+M) because of the possible adaptation of the assistance to the context but decreases 'manual usage' (-L) since the user has to adjust assistance parameters.

To be able to fulfill Table 6 automatically using more fundamental or partial knowledge is quite a challenging issue. A way to overcome this difficulty is to trust experts. Even if Table 6 was the most pertinent, it is not so clear how to aggregate such data and so how to select the most satisfying solutions. It is the purpose of this study case to illustrate hereafter how the method developed in section 4 can help designers make their choice rationally from Table 6.

Thanks to the qualitative analysis developed in Section 4, we can now compute the impacts of the various ASDS on the satisfaction level on criteria.

For instance, let's consider the particular ASDS defined by the 8_tuple $x=\left\{x_{1}: 3, x_{2}: 2, x_{3}: 2, x_{4}: 1, x_{5}: 1, x_{6}: 2, x_{7}: 2, x_{8}: 2\right\}$ (i.e. the gray case in Table 6), oDDPs $i, i \in\{1, . ., 7\}$ the oDDPs linked with the 'Mobility' criterion and oDDPs $i, i \in\{8, . ., 11\}$ the oDDPs linked with the 'User Friendliness' criterion.

- Applying expression (1) in assuming a pessimistic attitude, the impact of ASDS $x$ on oDDP6 (autonomy) is $(0, \mathrm{~L})$ and $(\mathrm{O}, \mathrm{H})$ on oDDP10 (manual usage) since :

$$
\begin{aligned}
& \min _{j} \delta_{6 j}^{+}=\min (L, M, M, L, M, M)=L ; \max _{j} \delta_{6 j}^{-}=\max (L, L)=L \\
& \text { so } \delta_{6, x}^{+1}=0 \text { and } \delta_{6, x}^{-1}=\max _{j} \delta_{6 j}^{-}=\mathrm{L} \text {; } \\
& \min _{j} \delta_{10 j}^{+}=0 ; \max _{j} \delta_{10 j}^{-}=\max (M, L, L, H, M, L)=H \\
& \text { so } \delta_{10, x}^{+1}=0 \text { and } \delta_{10, x}^{-1}=\max _{j} \delta_{10, j}^{-}=\mathrm{H} \text {; }
\end{aligned}
$$

- Applying expression (2) in assuming an optimistic attitude, the impact of ASDS $x$ on oDDP6 (autonomy) is $(\mathrm{M}, 0)$ and $(0, \mathrm{H})$ on oDDP10 (manual usage) since :

$$
\begin{gathered}
\left(\max _{j} \delta_{6 j}^{+}=\max (L, M, M, L, M, M)=M\right)>\left(\max _{j} \delta_{6 j}^{-}=\max (L, L)=L\right) \\
\text { so } \delta_{6, x}^{+2}=\mathrm{M} \text { and } \delta_{6, x}^{-2}=0 \\
\left(\max _{j} \delta_{10 j}^{+}=0\right)<\underset{j}{<}\left(\max _{10 j}^{-}=\max (M, L, L, H, M, L)=H\right) \\
\text { so } \delta_{10, x}^{+2}=0 \text { and } \delta_{10, x}^{-2}=\mathrm{H}
\end{gathered}
$$

According to (3) and with the optimistic attitude assumption:

- the impact of ASDS $x$ on all oDDPs relative to 'Mobility' is:

$$
\delta_{M x}^{2}=\left(\delta_{M x}^{+, 2}, \delta_{M x}^{-, 2}\right)=\left(\min _{i \in\{1, . .7\}}\left(\delta_{i, x}^{+, 2}\right), \max _{i \in\{1, .7\}}\left(\delta_{i, x}^{-, 2}\right)\right)=(0, L)
$$

- the impact of ASDS $x$ on all oDDPs relative to 'User Friendliness' with $\mathrm{U} 1=-\mathrm{H}, \mathrm{U} 2=-\mathrm{L}, \mathrm{U} 3=-\mathrm{H}$ (Table 6) is:

$$
\delta_{U F x}^{2}=\left(\delta_{U F x}^{+, 2}, \delta_{U F x}^{-, 2}\right)=\left(\min _{i \in\{8, . ., 11\}}\left(\delta_{i, x}^{+, 2}\right), \max _{i \in\{8, . ., 11\}}\left(\delta_{i, x}^{-, 2}\right)\right)=(0, H)
$$


Under the optimistic attitude assumption , the most promising ASDS relative to 'Mobility' on one side and 'User friendliness' on the other side can be determined by applying expression (4). Out of the total 576 ASDS, 20 are the most promising as regards 'Mobility' (the colored iDDPs choices on line 'Mobility' in Table 7) and 8 are the most promising as regards 'User friendliness' (the colored iDDPs choices on line 'User friendliness' in Table 7).

\begin{tabular}{|c|c|c|c|c|c|c|c|c|c|c|}
\hline \multirow{3}{*}{$\begin{array}{c}\text { Input DDP } \\
\text { Choices }\end{array}$} & \multicolumn{2}{|c|}{ iDDP5 } & \multicolumn{2}{|c|}{ iDDP6 } & \multicolumn{2}{|c|}{ iDDP7 } & \multicolumn{2}{|c|}{ iDDP8 } & & \\
\hline & \multicolumn{2}{|c|}{$\begin{array}{l}\text { Electronics } \\
\text { board location }\end{array}$} & \multicolumn{2}{|c|}{ Control type } & \multicolumn{2}{|c|}{ Setting } & \multicolumn{2}{|c|}{ Feedback to user } & & \\
\hline & $\begin{array}{l}\text { on chair } \\
\text { (1) }\end{array}$ & $\begin{array}{c}\text { on wheel } \\
(2)\end{array}$ & $\begin{array}{c}\text { smart } \\
\text { control } \\
(1)\end{array}$ & $\begin{array}{c}\text { reflex } \\
\text { control } \\
(2)\end{array}$ & $\begin{array}{c}\text { no tuning } \\
\text { possibilty } \\
(1)\end{array}$ & $\begin{array}{c}\text { tuning } \\
\text { posibility } \\
(2)\end{array}$ & $\begin{array}{c}\text { no } \\
\text { feedback } \\
(1)\end{array}$ & $\begin{array}{c}\text { feedback } \\
\text { to user } \\
\text { (2) }\end{array}$ & & \\
\hline \multirow{2}{*}{\multicolumn{11}{|c|}{ Mobility }} \\
\hline & & & & & & & & & & \\
\hline \multicolumn{11}{|l|}{ Jser Friendlines } \\
\hline \multicolumn{11}{|l|}{ Employability } \\
\hline \multirow[b]{2}{*}{ Input DDP } & \multicolumn{3}{|c|}{ iDDP1 } & \multicolumn{3}{|c|}{ iDDP2 } & \multicolumn{2}{|c|}{ iDDP3 } & \multicolumn{2}{|c|}{ iDDP4 } \\
\hline & \multicolumn{3}{|c|}{ Reduction type } & \multicolumn{3}{|c|}{$\begin{array}{l}\text { Motors } \\
\text { number }\end{array}$} & \multicolumn{2}{|c|}{ Battery location } & \multicolumn{2}{|c|}{$\begin{array}{c}\text { Wheel structure } \\
\text { rigidity }\end{array}$} \\
\hline Choices & $\begin{array}{l}\text { gear on } \\
\text { motor (1) }\end{array}$ & $\begin{array}{l}\text { rack and } \\
\text { pinion (2) }\end{array}$ & mixed (3) & $\begin{array}{c}1 \text { motor } \\
(1)\end{array}$ & $\begin{array}{c}3 \text { motors } \\
(2)\end{array}$ & $\begin{array}{c}>3 \\
\text { motors } \\
(3)\end{array}$ & \begin{tabular}{|c|} 
package \\
on wheel \\
$(1)$
\end{tabular} & \begin{tabular}{|c|} 
package \\
on chair \\
$(2)$ \\
\end{tabular} & high (1) & $\begin{array}{r}\text { some } \\
\text { flexibiity } \\
(2)\end{array}$ \\
\hline \multirow{2}{*}{\multicolumn{11}{|c|}{ Mobility }} \\
\hline & & & & & & & & & & \\
\hline \multicolumn{11}{|l|}{ Jser Friendlines } \\
\hline Employability & & & & & & & & & & \\
\hline
\end{tabular}

TABLE 7: Most promising choices (coloured areas) for Mobility, UserFriendliness, and Employability (darkest area on line Employability ensure best satisfaction on the three criteria)

Ultimately, under the optimistic attitude assumption, most promising ASDS for 'Employability' are those that maximize the minimum positive impacts of ASDS on both oDDPs relative to 'Mobility' and to 'User friendliness' provided such a minimum is nonzero or, otherwise, that minimize the maximum negative impacts of ASDS on oDDPs relative to (Mobility' and 'User Friendliness').

Therefore, by computing the following:

$$
\left\{\begin{array}{c}
\underset{x}{\arg \max }\left(\min _{x}\left(\delta_{M x}^{+, 2}, \delta_{U F x}^{+, 2}\right)\right) \text { if } \exists x: \min _{x}\left(\delta_{M x}^{+, 2}, \delta_{U F x}^{+, 2}\right) \neq 0 \\
\underset{x}{\arg \min }\left(\max _{x}\left(\delta_{M x}^{-, 2}, \delta_{U F x}^{-, 2}\right)\right): \text { otherwise }
\end{array}\right.
$$

The so found 16/576 most promising ASDS are shown in Table 7 (colored iDDPs choices on line 'Employability'). In this particular case, the number 16 is obtained because whatever the choices of iDDP6, iDDP7, iDDP8 ( $2 \times 2 \times 2$ possibilities) these solutions are the most satisfying. It can be noted that the set of ASDS with a higher degree of utility for the 'Employability' criterion is larger than the intersection of the set of ASDS with a higher degree of utility for the 'Mobility' criterion and the set of ASDS with a higher degree of utility for the 'User Friendliness' criterion.

From the sixteen most promising ASDS for the 'Employability' criterion, four are also the most promising for the 'Mobility' and 'User-friendliness' criteria. They are those corresponding to (the darkest iDDPs choices): mixed reduction type (gearbox and rack and pinion), 3 motors on each wheel, battery and electronics boards on wheel, not too a rigid structure, feedback provided to the user. As regards control type and tuning facilities choices remain opened. 
The results of table 7 do not show any incoherence, and illustrate how the qualitative method proposed in Section 4 can be applied on a mechatronic study case. Even if Table 6 could have been fulfilled by experts in different disciplines, the proposed qualitative evaluation method allows to synthetize their advices in a rational, reproducible and traceable way.

\section{$\underline{\text { Remarks: }}$}

- It must be clarified that these results depend on any translation of the impact value on the PS or the NS scale. As a matter of fact, translating these values means that the current state $y$ of the oDDP has changed. For instance, any component added to the product adversely affects the total wheelchair weight (so no choice could feature a positive impact!). In assuming a nonzero total weight however, choosing between the two solutions may modify this weight either positively or negatively.

- As seen in Section 2, a sensitivity analysis must be performed. In pursuing this goal, we have not yet investigated any approaches other than that consisting of testing an array of impact values. As an example, changing $U 2$ values from $U 2=-L$ to $U 2=-M$ does not change the most promising ASDS for 'Employability', although changing $\mathrm{U} 2=-\mathrm{M}$ to $\mathrm{U} 2=-\mathrm{H}$ actually eliminates all ASDS. It is obvious that the experts need to be truly trusted professionals.

- For a more flexible attitude, we could assign a weighting distribution to the oDDPs related to the customer preference [39].

\section{Conclusion and outlook}

Mechatronic design is an interdisciplinary activity that always tends to integrate more functionality into geometrically-constrained products. What makes mechatronic design quite specific is the need for an interdisciplinary problem solving approach at the earliest of the preliminary design stages. The role of the evaluation process therefore is to support designers each time engineering choices need to be made or justified. However it is often difficult to anticipate, during these earlier stages, the consequences of design decisions on the effectiveness of such complex products. Thus the designers should be able to evaluate the system properties that result from design changes in the functional and physical architectures of the system even when models of the system are still coarse. The proposed evaluation method aims at achieving this objective.

By adopting the MBSE framework in Systems Engineering, we have proposed a meta-model of evaluation so that designers from different technical backgrounds can share a common vision of the evaluation process. Based on the relationships exhibited by such a meta-model, we have also proposed a protocol for identifying the potential impact relations of design choices on future product performance. Depending on the accuracy and precision of the models introduced to advance the design, a quantitative or qualitative analysis of such impacts must be performed. During the conceptual design phase, given that the data are quite uncertain or imprecise, we have extended an original qualitative impact analysis in order to detect the most promising alternative system design solutions. Our proposals have been illustrated through the design of a mechatronic system.

The proposals we've presented are complementary to current design approaches, which focus on ensuring that mechatronic products are well-designed, since our scope is more oriented around design validation (to satisfy stakeholder requirements) than design verification (to follow the best design practices in ensuring highest product quality). Both of these focuses are indeed necessary.

Although the design of an electrical assistance for a wheelchair is a typical example of mechatronic interdisciplinary design on which we successively applied our proposals, the method is not necessarily unique to mechatronics product development but may be applied in other interdisciplinary field of engineering. 
In addition to incorporating most of the recent research on decision theory (in the aim of increasing the level of trust designers can show in the evaluation process), our research has been intended to improve the consistency between successive and more refined evaluation results through conducting the SE process in successive layers, thus yielding detailed insight into the design and taking advantage of both inter and intra SE-level traceability relations.

\section{References}

[1] F. Hehenberger et al., Hierarchical design models in the mechatronic product development process of synchronous machines, Mechatonics, Vol 20, issue 8, 2010, pp. 864-875.

[2] Nasa System Engineering Handbook, 2007, Nasa SP-2007-6105, Rev 01.

[3] J.M.Torry-Smith et al., , Mechatronic design - still a considerable challenge, ASME IDECT/CIE, Washington, August 28-31, 2011, 12p.

[4] T. Tomyama, P. Gu, Y. Jin, Lutters D., Kind Ch. and Kimura F., Design methodologies: Industrial and educational applications, CIRP Annals-Manufacturing Technology, 2009, 543-565.

[5] D. Sharman, A. Yassine, Characterizing Complex Products Architectures, Systems Engineering, 7(1), pp.3560, 2004.

[6] M.E. Sosa, S.D. Eppinger and C.M. Rowles, A network approach to define modularity of components in complex products, Trans. AMSE 129, 2007, 1118-1129.

[7] A. Yassine, An Introduction to Modeling and Analyzing Complex Product Development Processes Using the Design Structure Matrix (DSM) Method (http://users.ipfw.edu/reddpv01/DSMTutorial.pdf [last visited 2011-07$12])$.

[8] B.S. Blanchard, W. J. Fabricky, Systems Engineering and Analysis, Fifth Edition, Prentice Hall Series, Industrial And Systems Engineering, 2011, $800 \mathrm{p}$

[9] J. Gausemeier , S.,Moehringer, VDI 2206 - a new guideline for the design of mechatronic system, Mechatronic Systems 2002: A Proceedings Volume from the 2nd Ifac Conference Berkeley, California, Usa, 911, 2002, p. $785-790$.

[10] J.S. Gero, U. Kannengiesser, Towards a situated Function-Behaviour-Structure framework as the basis of a theory of designing, 2000, Vol. 1. No 5.

[11] Umeda Y, Ishii M, Yoshioka M, Tomiyama T (1996) Supporting Conceptual Design Based on the Function-Behavior-State Modeler. Artificial Intelligence for Engineering Design Analysis and Manufacturing, 1996, p275-288.

[12] H. Komoto, T. Tomiyama, A system architecting tool for mechatronic systems design, Cirp Annals, Manufacturing Technology, 2010, p. 171-174.

[13] N.P. Suh, The principles of design. New York: Oxford University Press, 1990.

[14] J. Beom-Seon et al., Axiomatic design approach for marine design problems, Marine Structures, 15, 2002, p. $35-56$.

[15] V.C. Moulianitis, N.A. Aspragathos and A.J. Dentsoras, A model for concept evaluation in design- an application to mechatronics designof robot grippers, Mechatronics, 14, 2004, p. 599-622.

[16] ISO/IEC, IEEE Standards 15288.2008, Systems engineering - System life cycle processes (2nd edition), 2008.

[17] BKCASE Project, System Engineering Book of Knowledge, SEBoK v1.0, 2012, voir http://www.sebokwiki.org/ 
[18] INCOSE, System Engineering (SE) Handbook Working Group, System Engineering Handbook, A Guide For System Life Cycle Processes And Activities, Version 3.2.1, 2011,INCOSE TP 2003 002 03.2.

[19] R. Kopach-Konrad et al., Applying Systems Engineering Principles in Improving Health Care Delivery, Journal of General Internal Medicine, Volume 22, Supplement 3, 2007, pp. 431-437.

[20] System Engineering guide book for transportation systems, California Department of Transportation, Division of research and transportation, 2007.

[21] RG AERO 00077 : Programme management - Guide for the management of Systems Engineering, BNAE Bureau de Normalisation de l'Aéronautique et de l'Espace, 2005.

[22] M. W. Maier, E. Rechtin, The Art of System Architecting, Third Edition, CRC Press, 2009, 472 p.

[23] J. Fülöp, Introduction to Decision Making Methods, Laboratory of Operations Research and Decision Systems, Computer and Automation Institute, Hungarian Academy of Sciences, 2005, 15p.

[24] R. L. Keeney, G. L. Lilien, New Industrial Product Design and Evaluation Using Multiattribute Value Analysis, J. Prod InnovManag, 1987, pp. 185-198.

[25] T.L. Saaty, L.T. Tranb, On the invalidity of fuzzifying numerical judgments in the Analytic Hierarchy Process, Mathematical and Computer Modelling,46, 2007, pp. 962-975.

[26] B. Roy, The outranking approach and the foundations of electre methods, Theory and Decision, Volume 31, 1991, pp. 49-73.

[27] K. L. Wood, E. K Antonsson, Computations with imprecise parameters in engineering design: background and theory, ASME Journal of Mechanisms, Transmissions, and Automation in Design, Volume 111, Number 4, 1989, pp. 616-625.

[28] INCOSE, Survey of Model-Based Systems Engineering (MBSE) Methodologies, INCOSE-TD-2007-00301, Version/Revision: B, 10 June 2008.

[29] O. L. de Weck, A. M.Ross and D. H. Rhodes, Investigating Relationships and Semantic Sets amongst System Lifecycle Properties (Ilities), third International Engineering Systems Symposium CESUN 2012, Delft University of Technology, 18-20 June, 2012.

[30] Berenbach B., An Introduction to Project and Product Traceability, Incoce Insight, December, Volume 14 Issue 4, 2011.

[31] S.F. Königs, G. Beier, A. and Figge, R. Stark, Traceability in Systems Engineering - Review of industrial practices, State-of-the-art technologies and new research solutions, Advanced Engineering Informatics, 2012, pp. 924-940.

[32] Vitech Corporation, A primer for Model Based Systems Engineering, 2011.

[33] T.Quirante. Y. Ledoux and P. Sebastian, Multiobjective optimization including design robustness objectives for the embodiment design of a two-stage flash evaporator, IJIDEM 6 (1), 2012, 29-39.

[34] P.C. Fishburn, Utility Theory for Decision-Making. John Wiley \& Sons, New York, 1970.

[35] P.C. Fishburn, The foundations of expected utility. Dordrecht: Reidel, 1982.

[36] R.L. Keeney, H. Raiffa, Decisions with Multiple Objectives Preferences and Value Tradeoffs. Cambridge University Press, 1976

[37] Imoussaten, A., J. Montmain, F. Trousset, and Ch. Labreuche, Multi-criteria improvement of options. In Proceedings of the 7th conference of the European Society for Fuzzy Logic and Technology (EUSFLAT-2011) 
and LFA-2011, Advances in Intelligent Systems Research, pages 1030 - 1037, Aix-les-Bains, France, 2011, Atlantis Press.

[38] P. Giorgini, J. Mylopoulos J., E. Nicchiarelli and R. Sebastiani, Reasoningwith Goal Models, 21st International Conference on Conceptual Modeling (ER02), Tampere (Finland), 2002,167-181.

[39] J. Montmain, V. Clivillé, L. Berrah, and G. Mauris, Preference and causal fuzzymodels for manager's decision aiding in industrial performance improvement, FUZZY-IEEE, 2010.

[40] CERAH, Spécifications Techniques Vehicules pour Handicaps Physiques, Titre IV de la LPPR, 2010.

[41] M. Lô., P. Couturier, Needs for tracing the consequences of decisions in mechatronics design, Proceedings of the ASME2012 11th Biennial Conf. On Engineering Systems Design and Analysis, Nantes, 2012, July 2-4. 